\title{
Quality Measurement in Cancer Care: A Review and Endorsement of High-Impact Measures and Concepts
}

Thomas A. D'Amico, MDa; Lindsey A.M. Bandini, MPH; Alan Balch, PhDc; Al B. Benson III, MDd; Stephen B. Edge, MDe; C. Lyn Fitzgerald, MJb; Robert J. Green, MDf; Wui-Jin Koh, MDb; Michael Kolodziej, MDg; Shaji Kumar, MD'; Neal J. Meropol, MDf; James L. Mohler, MDe; David Pfister, MDi; Ronald S. Walters, MD, MBA, MHA, MSj; and Robert W. Carlson, MD

\section{ABSTRACT}

Although oncology care has evolved, outcome assessment remains a key challenge. Outcome measurement requires identification and adoption of a succinct list of metrics indicative of high-quality cancer care for use within and across healthcare systems. NCCN established an advisory committee, the NCCN Quality and Outcomes Committee, consisting of provider experts from NCCN Member Institutions and other stakeholders, including payers and patient advocacy, community oncology, and health information technology representatives, to review the existing quality landscape and identify contemporary, relevant cancer quality and outcomes measures by reevaluating validated measures for endorsement and proposing new measure concepts to fill crucial gaps. This manuscript reports on 22 measures and concepts; 15 that align with existing measures and 7 that are new.

J Natl Compr Canc Netw 2020;18(3):250-259 doi: $10.6004 /$ jnccn.2020.7536

\section{Background}

The modern era of quality measurement and reporting in healthcare began in the 1980s when The Joint Commission on Accreditation of Healthcare Organizations (JCAHO) began the systematic evaluation of hospitals. Until the 1990s, cancer care quality was addressed primarily through setting standards for organizational structure through JCAHO and the American College of Surgeons (ACS) Commission on Cancer (CoC) accreditation programs. ${ }^{1}$ In 1999, the Institute of Medicine (IOM) established the currently accepted framework for quality measurement in oncology, in part based on the structure, process, and outcome model for assessment of healthcare quality defined in the 1960s by Avedis Donabedian. ${ }^{2}$ Shortly thereafter, the National Quality Forum (NQF) initiated a formal multistakeholder process for identification and validation of quality measures in oncology. ${ }^{3}$

The recent focus on value in cancer care and the establishment of alternative payment models has renewed interest in the development of a more standardized, contemporary, clinically relevant, easily implemented, and broadly applicable set of quality and outcome measures. The Centers for Medicare \& Medicaid Services (CMS) has solicited recommendations for quality measures to include within programs such as the Merit-based Incentive Payment System (MIPS) Value Pathways, the next step in the MIPS program, ${ }^{4}$ and the new Oncology Care First (OCF) Model. ${ }^{5}$ Commercial payers have shown equal interest in determining which existing metrics are of high value, and where gaps in good measures exist. ${ }^{6}$

Most measures to date focus on key processes of care that are evidence-based and intended to improve outcomes. Maintaining a set of relevant high-impact quality measures has proved challenging due to many factors. Rapid innovation in oncology practice, including national changes to coverage reimbursement models, advancements in information technology, and the introduction of practice-changing treatments, have outpaced the historically slow measure development process. 
Additionally, measures are difficult to evaluate in randomized clinical trials, and therefore many measures rely on best practices based on historical consensus. As a result, many of the leading quality measurement programs have begun to reevaluate their strategies for measuring high-quality cancer care within and across healthcare systems, with the goal of identifying appropriate measures that are feasible, offer the opportunity to document quality improvement, and can be extracted using available and reliable data for ease of evaluation and reporting. ${ }^{7,8}$

NCCN established the NCCN Quality and Outcomes Committee (the Committee), consisting of experts from NCCN Member Institutions and other stakeholders, including payers and patient advocacy, community oncology, and health information technology representatives, to review the existing quality landscape and identify contemporary, relevant cancer quality and outcomes measures by both reevaluating current validated measures and proposing potential new measure concepts to fill crucial gaps.

This article reports on the methods used to derive a priority list of measures and concepts, including those that closely align with existing measures and those that represent novel constructs. The Committee aimed to provide critical support for inclusion of existing measures where appropriate and advance novel measure concepts where necessary in quality programs or value-based models. Additionally, the Committee aimed to lend their collective voice to encourage better alignment across value-based programs in oncology to reduce clinical and administrative burden and promote more meaningful benchmarking across practices.

\section{Methods}

The Committee initially met in 2016 to explore the oncology quality measurement landscape and prioritize areas of focus. Initial attention was directed toward the top 10 cancer groups by incidence in the United States: breast, lung, prostate, colorectal (CRC), leukemia and lymphoma, bladder, uterine, thyroid, kidney, and pancreas. ${ }^{9}$

The Committee discussed the potential impact of quality measurement in each disease group. Consensus was reached on a plan to focus on the 4 most common cancer types and cross-cutting measures that span multiple cancer types for greatest impact. Breast and lung ranked highest for potential impact, followed closely by CRC and prostate. Applicable existing measures were reviewed based on importance, supporting evidence, opportunity for improvement, and ease of measurement using a modified Delphi process in conjunction with telephonic web-based meetings monthly and at least 2 in-person meetings annually (Figure 1).
A comprehensive list of 528 existing oncologyspecific quality measures or concepts was curated from Committee discussions and from oncologyspecific professional organizations, including the NQF and the National Quality Measure Clearinghouse (NQMC). In this article, measures are defined as the codifications, with or without quantifiable specifications, that have been developed by recognized process organizations. Concepts refer to the codifications that are novel in origin that the Committee are evaluating for further potential development. Both groups of measures and concepts were assigned thematic labels, referred to as measurement streams, based on the Committee's priorities. The 3 streams were patient experience, treatment team, and evidence-based concordance measures. The third stream of measures addressed specific nodes within guidelines developed by NCCN, ASCO, and other professional organizations, and were of interest to multiple stakeholders, including payers who had emphasized the importance of treatment decisions grounded in scientific evidence. Evaluations of these evidence-based concordance measures were led by NCCN Guidelines Panel Members and focused on the overall importance of the specific measurement nodes in improving patient outcomes and quality of care.

Measures and concepts underwent 3 rounds of review. Four disease-specific subcommittees, comprising disease experts from the committee at-large and additional experts from NCCN Guidelines Panels, were convened to perform the initial review of measures specific to breast, lung, CRC, and prostate cancers, and evaluate which guideline decision nodes would likely impact patient outcomes and quality of care. The crosscutting measures and measures that advanced to subsequent rounds were reviewed by the overall Committee.

Measures and concepts were evaluated according to the following criteria: importance, supporting evidence, opportunity for improvement, and ease of measurement. Each round consisted of a discussion followed by an online vote for each measure or concept. The firstround survey consisted of a binary yes/no response indicating support for including a specific measure, whereas subsequent rounds ranked enthusiasm on a Likert scale (high, moderate, and low) based on each of the criteria.

Throughout the process, the Committee engaged in dialogue with key stakeholders to gather additional insight and to identify areas of alignment or dissent. Healthcare technology partners provided insight into the clinical and technical challenges of collecting specific measures, specifically identifying those that would require human abstraction versus information regularly collected in a structured data field in electronic 


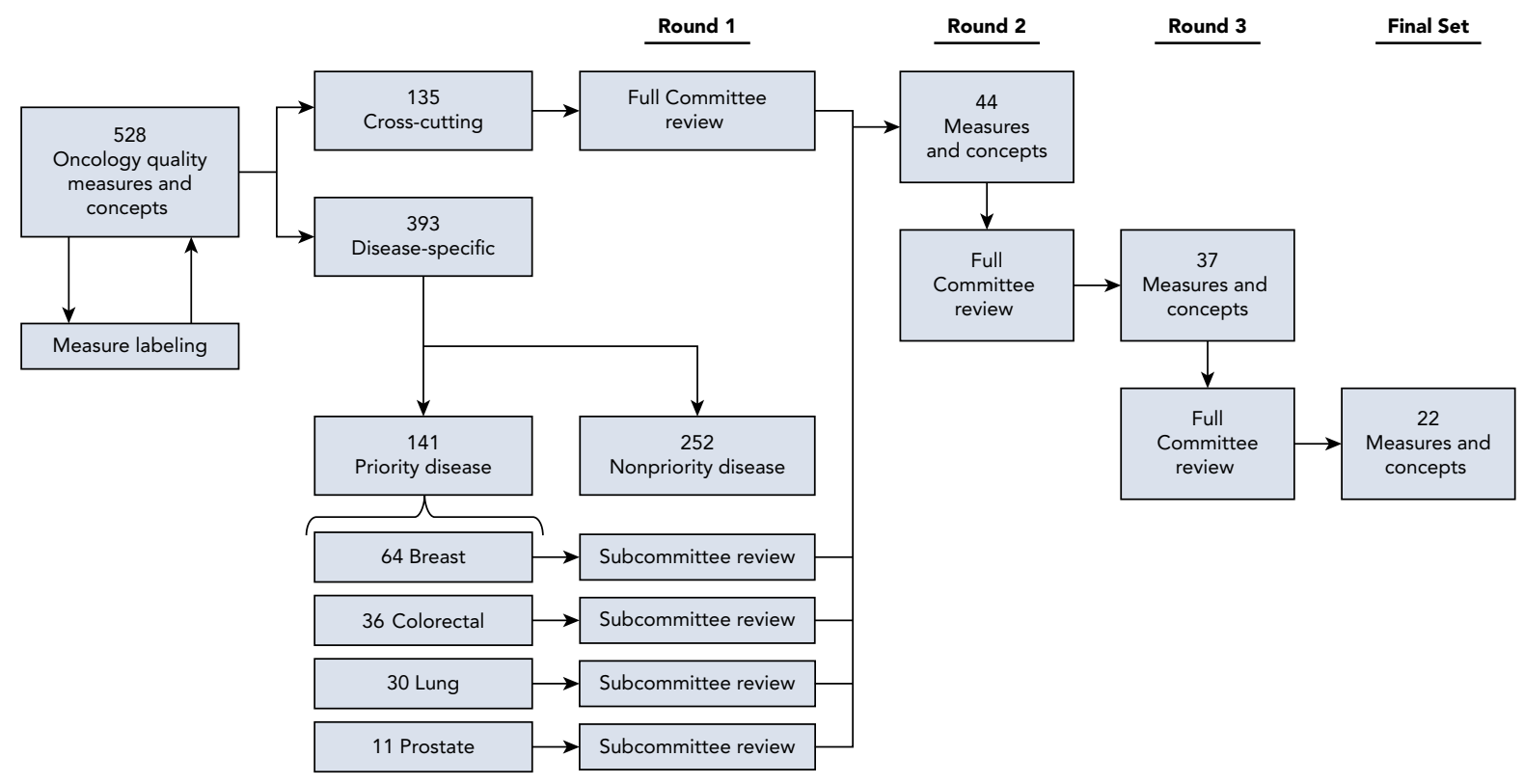

Figure 1. Flowchart of the quality measure and concept review process.

health records. NCCN Member Institutions, community providers, and commercial payers were asked to provide feedback on clinical utility, feasibility, and value of certain measures in practice. Finally, patient advocacy organizations (including disease-specific organizations for breast, CRC, prostate, and lung cancers) provided feedback on patient experience measures under consideration.

The resulting list included 22 measures relating to the concepts of interest: 7 cross-cutting measures, and 6 prostate, 5 CRC, 3 breast, and 1 lung cancer measures. The final list was compared with existing quality measures used by the various measurement programs to account for changes since project initiation.

Measures with reasonable alignment to existing measures were categorized for endorsement, whereas the novel constructs were identified as suitable for development.

\section{Results}

The Committee selected 15 quality measures for endorsement (Table 1), ${ }^{10-28}$ all with some alignment to measures developed or used by the CoC, Choosing Wisely, ASCO's Quality Oncology Practice Initiative (QOPI), MIPS, the Prospective Payment System (PPS)-Exempt Cancer Hospital Quality Reporting (PCHQR) Program, the Oncology Medical Home, the Center for Medicare \& Medicaid Innovation's (CMMI) Oncology Care Model (OCM), or The University of Texas MD Anderson Cancer Center. Some measures were reviewed by NQF, but endorsement was not a requirement for inclusion on this list. Specific measures aligning with the selected concepts were outlined; however, measure specifications were not reviewed thoroughly, and therefore the Committee did not select preferred measures.

Seven measure concepts selected by the Committee lack reasonable alignment to existing measures and are therefore highlighted as opportunities for development (Table 2). These measure concepts represent critical gaps in measurement for breast, CRC, and prostate cancers.

A summary of themes and important considerations for measures within each disease area are listed below.

\section{Breast}

Selected measures in breast cancer represent important diagnostic and treatment decisions throughout the care continuum with proven impacts on patient outcomes.

\section{Radiation With $\geq 4$ Positive Nodes}

Risk of local and regional recurrence is elevated substantially in patients with breast cancer and multiple involved axillary lymph nodes. Meta-analysis by the Early Breast Cancer Trialists' Collaborative Group (EBCTCG) showed improved outcomes with radiation to the chest wall and regional lymph nodes after mastectomy with at least a level II axillary dissection with $\geq 4$ positive nodes and following receipt of adjuvant systemic therapy. ${ }^{29}$ Locoregional recurrence occurred in $13 \%$ of patients who received radiation versus $32 \%$ who did not (log-rank, 2 -sided $P<.00001)$. Overall mortality after 20 years was 


\section{Table 1. Quality Measures Selected by the NCCN Quality and Outcomes Committee}

\begin{tabular}{|c|c|c|c|}
\hline Disease & Measurement Stream & Measure & Similar Measures \\
\hline Breast & Evidence-based concordance measure & $\begin{array}{l}\text { Patients with } \mathrm{M} 0 \text { disease, and } \geq 4 \text { involved } \\
\text { axillary lymph nodes, receive breast/chest wall } \\
\text { plus regional lymph irradiation as part of their } \\
\text { treatment }\end{array}$ & $\mathrm{CoC}^{10}$ \\
\hline Breast & Evidence-based concordance measure & $\begin{array}{l}\text { Tumor markers are not performed during the } \\
\text { period of follow-up surveillance for those who } \\
\text { have completed breast cancer treatment with } \\
\text { curative intent }\end{array}$ & Choosing Wisely, ${ }^{11}$ QOPI $(62 c 1 \& 62 c 2)^{12}$ \\
\hline Colorectal & Evidence-based concordance measure & $\begin{array}{l}\text { For patients with resected pathologic stage II } \\
\text { and III colorectal cancer in surveillance, } \\
\text { carcinoembryonic antigen is performed at least } \\
\text { every } 6 \text { months for } 5 \text { years }\end{array}$ & QOPI $(66)^{12}$ \\
\hline Colorectal & Evidence-based concordance measure & $\begin{array}{l}\text { Patients with rectal cancer are staged with a CT } \\
\text { scan of chest, abdomen, and pelvis and pelvic } \\
\text { MRI with contrast or endorectal ultrasound } \\
\text { before surgery }\end{array}$ & QOPI $(78)^{12}$ \\
\hline Cross-cancer & Evidence-based concordance measure & $\begin{array}{l}\text { Proportion admitted to the intensive care unit } \\
\text { in the last } 30 \text { days of life }\end{array}$ & $\begin{array}{l}\text { QOPI (49icu), },^{12} \text { MIPS (455), }{ }^{13} \text { PCHQR } \\
\left(\text { EOL-ICU), }{ }^{14} \text { NOF (0213) }{ }^{15}\right.\end{array}$ \\
\hline Cross-cancer & Evidence-based concordance measure & $\begin{array}{l}\text { Performance status documented prior to } \\
\text { initiating chemotherapy regimen }\end{array}$ & QOPI (13aa) $)^{12}$ \\
\hline Cross-cancer & Patient experience measure & $\begin{array}{l}\text { Patients are offered smoking cessation } \\
\text { counseling if current smoker }\end{array}$ & QOPI (22aa),,$^{12}$ MIPS $(226),{ }^{16}$ NOF $(0028)^{17}$ \\
\hline Cross-cancer & Treatment team & $\begin{array}{l}\text { Proportion receiving chemotherapy in the last } \\
14 \text { days of life }\end{array}$ & $\begin{array}{l}\text { QOPI (48), },^{12} \text { MIPS }(453),{ }^{18} \mathrm{PCHOR} \\
\left(\text { EOL-Chemo), }{ }^{14} \text { NQF }(0210)^{19}\right.\end{array}$ \\
\hline Cross-cancer & Treatment team & $\begin{array}{l}\text { Chemotherapy given within } 30 \text { days of end of } \\
\text { life }\end{array}$ & Oncology Medical Home $(15)^{20}$ \\
\hline Cross-cancer & Treatment team & Cancer stage documented & QOPI (2), ${ }^{12}$ NQF $(0386)^{21}$ \\
\hline Cross-cancer & Treatment team & $\begin{array}{l}\text { Proportion dying from cancer in an acute care } \\
\text { setting }\end{array}$ & NQF $(0214)^{22}$ \\
\hline Lung & Evidence-based concordance measure & $\begin{array}{l}\text { Palliative care consult is offered to patients } \\
\text { with metastatic non-small cell lung cancer } \\
\text { within } 8 \text { weeks of diagnosis }\end{array}$ & QOPI (43), ${ }^{12} \mathrm{NQF}(0215)^{23}$ \\
\hline Prostate & Evidence-based concordance measure & $\begin{array}{l}\text { Patients in the high-risk or very high-risk } \\
\text { prostate cancer groups, who receive radiation } \\
\text { therapy, receive ADT }\end{array}$ & $\begin{array}{l}\text { QOPI (117), } 12 \text { OCM (OCM-7), }{ }^{24} \text { PCHQR } \\
(\mathrm{PCH}-17),{ }^{15} \mathrm{NQF}(0390)^{25}\end{array}$ \\
\hline Prostate & Patient experience measure & $\begin{array}{l}\text { All patients treated with surgery or radiation } \\
\text { for localized prostate cancer should be } \\
\text { assessed for urinary incontinence and erectile } \\
\text { dysfunction with tools, such as the UCLA } \\
\text { Prostate Cancer Index questions and the } \\
\text { Sexual Health Inventory for Men }\end{array}$ & MD Anderson (CMS 5656 and 5657) 26,27 \\
\hline Prostate & Treatment team & $\begin{array}{l}\text { Prostate-specific antigen has been measured in } \\
\text { the last } 12 \text { months to monitor disease } \\
\text { recurrence in patients with prostate cancer }\end{array}$ & NQF $(0625)^{28}$ \\
\hline
\end{tabular}

Abbreviations: CMS, Centers for Medicare \& Medicaid Services; CoC, Commission on Cancer; EOL-Chemo, Proportion of Patients Who Died from Cancer Receiving Chemotherapy in the Last 14 Days of Life; EOL-ICU, Proportion of Patients Who Died from Cancer Admitted to the Intensive Care Unit, or ICU, in the Last 30-Days of Life; MIPS, Merit-based Incentive Payment System; NOF, National Quality Forum; OCM, Oncology Care Model; PCH, PPS-exempt cancer hospital; PCHOR, PPSExempt Cancer Hospital Quality Reporting; PPS, Prospective Payment System; QOPI, Quality Oncology Practice Initiative.

$70.7 \%$ with radiation therapy (RT) and $80 \%$ without RT $(2 P=.04)$. With breast-conserving surgery, radiation should also include regional nodal coverage when multiple nodes are positive. With $\geq 4$ positive nodes, the addition of regional nodal radiation to whole-breast RT with breast-conserving surgery or to the mastectomy site is recommended in major guidelines, including NCCN Guidelines with level I evidence. ${ }^{30}$ Recommendations for RT to the regional nodes also may impact patients with fewer involved nodes, but controversy remains, and therefore patients with 1 to 3 positive axially nodes were not included in this quality measure.

\section{Posttreatment Surveillance}

Markers of tumor in the serum or blood may help determine whether cancer has recurred in some cancer types. Unfortunately, no tumor marker in breast cancer is accurate or leads to outcome improvements for women who have completed therapy with curative intent (eg adjuvant therapy) and are undergoing surveillance. 


\section{Table 2. Quality Measure Concepts for Development}

\begin{tabular}{|lll|}
\hline Disease & Measurement Stream & Measure Concept \\
\hline Breast & Evidence-based concordance measure & $\begin{array}{l}\text { Cardiac function is assessed before starting and at least every } 4 \text { months during trastuzumab } \\
\text { therapy }\end{array}$ \\
\hline Colorectal & Evidence-based concordance measure & Adjuvant chemotherapy is not administered for patients with pathologic stage 1 colorectal cancer \\
Colorectal & Evidence-based concordance measure & PET scan is not performed for patients with locoregional colorectal cancer \\
\hline Colorectal & Evidence-based concordance measure & Patients with colon cancer are staged with CT scan of chest, abdomen, and pelvis before surgery \\
\hline Prostate & Evidence-based concordance measure & Patients with newly diagnosed prostate cancer have a risk group assigned \\
Prostate & Evidence-based concordance measure & $\begin{array}{l}\text { Patients in the very low-risk and low-risk prostate cancer groups do not receive androgen } \\
\text { deprivation therapy }\end{array}$ \\
\hline Prostate & Treatment team & $\begin{array}{l}\text { Patients with localized prostate cancer have a multidisciplinary evaluation or conference } \\
\text { (including urology, radiation oncology, pathology) before making a treatment decision }\end{array}$ \\
\hline
\end{tabular}

Previously, the hope was that assessing the level of epitopes of the MUC1 gene (CA 27.29 and 15-3) or the carcinoembryonic antigen (CEA) could reliably detect recurrence and lead to earlier and hence more effective therapy. However, evidence has shown that these markers are only elevated in a relatively small fraction of patients with recurrence and that there is a substantial false-positive rate. ${ }^{31}$ Therefore, these markers are of no overall value for breast cancer surveillance. For women undergoing surveillance after curative intent, these markers should not be routinely measured, particularly for nonmetastatic presentations. ${ }^{32}$ Serial testing of these markers may only be of value for women with metastatic breast cancer and initially elevated markers. Changes in the levels of these markers may provide early indication of response to systemic therapies. Therefore, this quality measure should be applied to women who have received curative-intent therapy for stage I-III breast cancer who undergo surveillance.

\section{Cardiac Monitoring With Trastuzumab Therapy}

The Committee selected one breast cancer-related concept related to treatment monitoring for patients treated with trastuzumab or a biosimilar. Discovery of the association of HER2 with aggressive breast cancer and the subsequent development of the monoclonal antibody trastuzumab dramatically improved the treatment outcome of patients with HER2-positive cancers. A significant toxicity associated with trastuzumab is the potential development of heart failure. Initial reports suggested rates of heart failure as high as $27 \% .^{33} \mathrm{~A}$ Cochrane systematic review in 2012 found that, in earlystage breast cancer, the relative risk of heart failure with trastuzumab was 5.1 compared with trastuzumab-free regimens, and that the development of heart failure was associated with a trastuzumab treatment interval of $>6$ months. ${ }^{34}$ The absolute risk of heart failure with trastuzumab was $2.4 \%$ compared with $0.4 \%$ in nontrastuzumab controls. A separate systematic review also confirmed increased cardiac risk in women treated with trastuzumab for metastatic cancer. ${ }^{35}$ Heart failure associated with trastuzumab appears reversible, unlike anthracycline-induced heart failure, and therefore warrants monitoring. Guidelines recommend cardiac function monitoring at 3- or 4-month intervals during therapy. ${ }^{29}$ However, recent evidence shows that $<50 \%$ of patients receive guideline-recommended monitoring. ${ }^{36}$

\section{Colorectal Cancer}

\section{Staging Evaluation in CRC}

The Committee emphasized the importance of appropriate care based on staging for patients with either colon or rectal cancer. Adequate staging for patients with potentially resectable colon cancer is essential and requires CT scans of the chest, abdomen, and pelvis with intravenous and oral contrast to maximize detection of potential metastatic disease. An alternative, if an abdominal or pelvic CT is inadequate or if CT with intravenous contrast is contraindicated, is abdominal/pelvic MRI with contrast plus a noncontrast chest CT. If metastatic disease is detected, individuals with potentially resectable metastatic lesions may still be candidates for curative-intent surgery. ${ }^{37}$

Similarly, the initial evaluation of patients with rectal cancer is critical to provide preoperative information and determine the clinical stage of disease. The clinical stage determines the primary treatment intervention, including curative-intent strategies for those with locoregional disease. A significant proportion of patients are treated with neoadjuvant therapy based on clinical stage, followed by surgical resection. CT scans of the chest and abdomen (or MRI of the abdomen) are required to assess evidence of distant metastases. Contrast-enhanced rectal (pelvis) MRI is the preferred imaging procedure to adequately assess local invasion and presence of regional lymph nodes. Endoscopic ultrasound is an alternative if MRI is contraindicated or rectal lesions 
appear superficial. ${ }^{38}$ Additionally, PET/CT scans are not recommended for the preoperative evaluation of patients with locoregional colon cancer, particularly because most PET/CT scans are performed without contrast and potentially lower resolution, and thus do not replace a contrast-enhanced diagnostic CT scan. A $\mathrm{PET} / \mathrm{CT}$ scan may be considered if abnormalities seen on CT or MRI are suspicious but inconclusive for metastatic disease, although subcentimeter lesions should not be evaluated by PET/CT because these are usually below the threshold of PET/CT detection. ${ }^{37,38}$

\section{Chemotherapy in Early-Stage CRC}

Treatment decisions, which include the selection or avoidance of certain treatments, rely on adequate staging. Overall survival of patients with resected stage I CRC is $>90 \%,^{39}$ and the addition of adjuvant chemotherapy for stage I CRC would provide minimal additional survival benefit while increasing the risk for toxicity. Therefore, patients with resected stage I CRC should not be treated with adjuvant chemotherapy. ${ }^{37}$

\section{Posttreatment Surveillance}

Recommendations for postacute surveillance of CRC rely on proper staging, and the following measure concepts were endorsed by the Committee. Data have shown that $80 \%$ of recurrences are diagnosed within the first 3 years after surgery and $95 \%$ are diagnosed by 5 years, thus justifying a limited time period for surveillance. ${ }^{40}$ One important purpose of surveillance is to identify early sites of isolated metastases for potential resection and attempted cure. The expanded number of regimens available to treat metastatic disease has improved survival significantly. The optimal surveillance strategy for stage II and III CRC remains controversial, but data have shown that CEA monitoring results in detection of more recurrences, including those that could be treated with curative intent, and in earlier detection of recurrent disease. ${ }^{37,38}$

\section{Lung Cancer}

\section{Early Palliative Care}

Use of palliative care has been shown to improve quality of life through reducing the burden of symptoms and improving patient function and mood. ${ }^{41}$ The value of palliative care in advanced lung cancer extends beyond comfort care, and has been shown to improve survival. ${ }^{42,43}$ The value of palliative care has been recognized widely by organizations such as ASCO, whose guidelines call for patients with advanced cancer to "receive dedicated palliative care services," and is a component of many existing and emerging value-based care programs. As an example, CMMIs $\mathrm{OCM}^{24}$ places an emphasis on integrating palliative care into routine cancer care, although palliative care consults were not called for specifically. The Committee agreed that inclusion of this measure is appropriate in these and other quality measurement programs for practices treating patients with advanced lung cancer.

\section{Prostate Cancer}

\section{RT and Androgen Deprivation Therapy}

Multiple randomized clinical trials have shown that definitive management of high-risk or very high-risk prostate cancer results in improved control or cure when RT is combined with neoadjuvant/concurrent/ adjuvant androgen deprivation therapy (ADT), which may last 18 to 36 months. ${ }^{44-46}$ The benefit of ADT may be due to early treatment of micrometastatic disease and cytoreduction of primary disease. Target volume reduction, when combined with advanced conformal radiation planning and delivery, improves outcomes by allowing selective dose escalation to residual tumor while maximizing sparing of adjacent critical normal structures, such as bladder and rectum. RT should not begin until at least the third month of ADT; earlier initiation of RT may mitigate the benefit of combined modality treatment by not allowing time for target volume reduction.

\section{Multidisciplinary Evaluation and Adverse Effect Assessment}

Patient focus is on cure as a result of RT or radical prostatectomy, but their quality of life may be affected adversely by urinary or fecal incontinence and erectile dysfunction. Because of variations in treatment risks and adverse effects, patients should be evaluated by a multidisciplinary team. Additionally, monitoring of prostatespecific antigen (PSA) level to evaluate oncologic control must be combined with validated instruments for adverse effect measurement to allow patients to evaluate radiation or surgical sequelae, the so-called trifecta. ${ }^{47}$

\section{PSA Monitoring}

PSA level correlates with tumor volume and should be measured at least annually in all men with prostate cancer. PSA level can be useful as part of a monitoring program for men on active surveillance. Monitoring the oncologic effectiveness of definitive treatment for localized prostate cancer is important in enabling men to make decisions about additional treatment of local recurrences. After radical prostatectomy, RT for local recurrences is more effective when initiated before a nadired PSA level increases to $0.5 \mathrm{ng} / \mathrm{mL}$. The NCCN definition for PSA recurrence is detectable PSA level followed by 2 subsequent increases. After RT (externalbeam, brachytherapy, or both), additional local treatments 
also may be used with curative intent or to provide longterm survival. The American Society for Radiation Oncology (ASTRO) definition of PSA-defined recurrence is PSA nadir $+2 \mathrm{ng} / \mathrm{mL}$. Possible therapies for local recurrence include radical prostatectomy, brachytherapy (if not used primarily), cryotherapy, high-intensity focused ultrasound, and experimental treatments. Treatments for local recurrences provide curative potential of approximately $50 \%$, but with increased chance of adverse effects, ${ }^{48}$ and are more likely to be successful if treatment is initiated sooner rather than later after PSA-defined recurrence has occurred. ${ }^{49}$ Because of the strength of data linking adherence to improved outcomes, the Committee feels strongly about the value of this measure, even without current NQF endorsement.

\section{Assigning Risk Groups}

Patients and physicians make better treatment decisions when prostate cancer is categorized into risk groups rather than just clinical stage. Most men with low-risk prostate cancer (NCCN very low, low, and favorable intermediate risk groups) should be offered, if not recommended, active surveillance to reduce overtreatment that complicates overdiagnosis. ${ }^{50}$ The relatively new Grade Group system, ${ }^{51}$ derived from the Gleason grading system, better aligns histologic grade and prognosis, and helps avoid men being frightened into treatment in response to concern over a Gleason score 6 cancer (scale of 2-10 when there are no 2-5 tumors) as opposed to a Grade Group 1 cancer (scale of 1-5). At the other end of the spectrum, patients with metastatic prostate cancer may benefit from the addition of chemotherapy for highvolume metastatic disease, compared with antiandrogens alone for low-volume metastatic disease.

\section{ADT and Low-Risk Prostate Cancer}

ADT is associated with adverse effects and significant expense and therefore should be used very infrequently, if at all, for patients with NCCN low-risk or very low-risk prostate cancer, especially those who are elderly (aged $\geq 65$ years). However, many patients with such low-risk disease are still treated with ADT. No overall survival benefit was demonstrated in 2 large cohort studies of $>80,000$ men treated with ADT..$^{52,53}$

\section{Cross-Cutting Cancer Measures}

Cross-cutting cancer measures are applicable to all phases of the cancer care continuum, but the currently selected measures and concepts mostly pertain to the beginning of this continuum and the end of the care phase.

\section{Recording Cancer Stage}

Once the cancer diagnosis is established, the stage is central to determining prognosis and treatment selection.
Stage is relevant to local, regional, and systemic treatment decisions, the timing of such decisions, and the appropriateness of such decisions, and stage serves as the starting branch point in treatment algorithms. The importance of staging information is recognized by ASCO and ACS and is included in QOPI and CoC certification processes, respectively. ${ }^{12,54}$ ASCO also sponsored this measure for endorsement by NQF as a core measure of quality in cancer care.

\section{Documenting Performance Status}

Performance status (PS) remains an important yet underdocumented part of oncologic assessment. ${ }^{55}$ PS is an indicator of prognosis, with a direct relationship to the likelihood of treatment response and benefit, and also predicts treatment toxicity and tolerance. This is particularly the case with the use of chemotherapy in treatment-refractory solid tumors, for which clinical trials documenting benefit beyond the first or second line of palliative drug therapy is typically lacking, and therefore providers may use other less-vetted agents. Practice guidelines often stratify treatment recommendations by PS; satisfactory PS is a ubiquitous eligibility in clinical trials, and therefore these trial results are less applicable to patients with a lower PS who would have been excluded from these studies. ${ }^{56}$

\section{Tobacco Cessation for Current Smokers}

Tobacco use is a risk factor for a spectrum of malignancies, and can affect the outcome (disease control and tolerance) of cancer treatments. ${ }^{57,58}$ Tobacco use adversely affects health in other domains, such as risk of vascular and pulmonary disease. Secondhand smoke also poses health risks. A diagnosis of cancer often represents a teachable moment for tobacco users, and effective interventions facilitate durable abstinence and behavior modification..$^{59,60}$ A tobacco cessation quality metric is endorsed by the NQF, MIPS, and QOPI.

\section{Treatment at End-of-Life}

Although exceptions do exist and patient preferences should be considered, chemotherapy given at the end of life, particularly for patients with a treatment-refractory malignancy and low PS, is usually futile oncologically and may adversely affect quality of life. ${ }^{61}$ Chemotherapy can distract from important goals-of-care discussions with the patient, their loved ones, and caregivers. ${ }^{62}$ The exact timeframe is disease- and situation-specific. An NQFendorsed measure assesses whether chemotherapy is administered in the last 14 days of life, whereas more recent work has focused on a 30-day window. The measured rate should not be $0 \%$, but high rates should be examined for dosing safety and clinical appropriateness. A similar NQF endorsed measure is the proportion of 
patients admitted to an intensive care unit in the last 30 days of life. The major purpose of both of these measures is to encourage a more active discussion of patient preferences and goals, the likelihood of achievement of meaningful life prolongation or palliation, and the toxicity and expense of treatment, guided by recommendations from the IOM in 2014. ${ }^{63}$ These end-of-life measures continue to be supported by numerous provider, patient advocacy, and healthcare system groups.

\section{Hospitalization at the End-of-Life}

The measure of percent of patients dying in an acute care setting has come under more discussion. ${ }^{61}$ The acute care setting is all that may be available in certain geographic and social settings. Patients with more symptoms may overwhelm the home care setting, especially when infrastructure and resources for compassionate and effective palliative care in the home are less than optimal. This last measure is no longer endorsed by NQF, but the Committee felt it is still an indicator of higher quality oncology care.

\section{Discussion}

As an alliance of 28 leading cancer centers, NCCN's mission is to improve and facilitate quality, effective, efficient, and accessible cancer care so that patients can live better lives. NCCN advances this mission through the creation and continuous update of clinical practice guidelines, which serve as a reliable source for patients, clinicians, payers, and other healthcare decision-makers around the world.

The Committee aimed to leverage their collective organizational experience and clinical expertise to evaluate points within care guidelines where attention to evidence is crucial to patient experience, outcome, or both. The Committee's review was not limited to specific quality measure developers, stewards, or programs, but was aimed to provide a nonpartisan evaluation of the entirety of the oncology quality measurement landscape.

This work represented an integral first step in aligning measures across models. It also may inspire measure developers to fill unmet needs, and cultivate collaboration among developers of similar measures. A thorough evaluation of measure specifications was outside the purview of this work; however, the Committee underscored the importance of simplifying the measurement space through aligning similar measures when possible. Alignment of measures across organizations will allow more efficient and focused use of resources, including reduction in variability of institutional measurement systems and/or the creation of streamlined technology solutions.

This endorsed list of measures contains universally appropriate metrics for evaluating quality of oncology care in a constantly changing health ecosystem. Consensus was achieved among disease experts and other key stakeholders that was intended to strengthen existing high-impact measures and encourage consideration of immediate implementation into quality measurement and/or value-based payment programs. These measures represented important opportunities for measurement backed by robust supporting evidence. The list was not exhaustive, but rather provided crucial support for fundamental measures most likely to improve patient outcomes in the near term. In selecting these measures, the Committee generally did not include measures for which there are very high rates of compliance, because use of these measures would have limited impact on quality improvement. In this vein, when evidence shows that the included measures are being met at a high rate, consideration should be given to replacing such measures with others where there is clear need for improvement.

The additional measure concepts meet the same criteria. They address areas with documented gaps within the measurement space and should be prioritized for development. These measures received priority ranking by the subcommittees for breast, CRC, lung, and prostate cancers, and the committee-at-large agreed on their potential high impact. This list should not be interpreted as a comprehensive list of gaps in oncology measurement, because it only represents those measures that are feasible for development and implementation in the short-term. Additional gaps were identified that included a significant need for better patient experience and cross-cancer metrics.

End-of-life care measures received significant attention among the cross-cutting cancer measures, which reflected the importance and unmet need. Existing endof-life measures mostly ranked high in ease of measurement, because the Committee and its technology representatives felt documentation could occur without major modifications to existing physician workflows or data collection tools, and many practices may be structured to collect this information. However, technical challenges in collecting and reporting end-of-life data have been documented, ${ }^{64,65}$ which should serve to inform the development of more complex measures that are universally applicable to any patient with cancer.

Patient experience measures also have many unanswered questions regarding collection, measurement, and utilization of standards. ${ }^{66}$ The Committee recognized a significant gap in measuring what truly matters to patients, and guidance is needed for overcoming these barriers. Technology has created some barriers, ${ }^{67}$ but also offers potential solutions, particularly when goals and specific needs align across patients, physicians, practices, payers, and policies; this has been 
demonstrated through rapid implementation of technology to support the early oncology value-based reimbursement models, such as MIPS and OCM.

The Committee recognized differences among practices that may result in limitations in applying or measuring certain quality metrics. For example, the Committee believes that, in most cases, the best palliative care intervention involves interdisciplinary teams with expertise and specialization in this space. However, these types of palliative care consultations are not available in all settings where patients with cancer receive care, and therefore may be delivered by a general oncologist or other practitioner. Although the Committee recognizes that some measures may be aspirational in certain situations, this list represents those that are attainable for most practices, and submits that these measures are appropriate for practice improvement initiatives.

Submitted September 19, 2019; accepted for publication January 10, 2020.

Disclosures: Dr. D'Amico has disclosed that he is a consultant for Scanlan. Dr. Benson has disclosed that he is a scientific advisor for Envision, Merck, Guardantt, Dava Onc, Bayer, Bristol-Myers Squibb, LSK, Association for ValueBased Cancer Care, Threabionic, Terumo, Lexicon, and Incyte. Drs. Green and Meropol have disclosed that they are employees of Flatiron Health, Inc., an independent subsidiary of the Roche Group, and have equity interests in Roche. Dr. Kolodziej has disclosed that he hold stock in CVS. Dr. Kumar has disclosed that he is a scientific advisor for Adaptive Technology and Oncopeptides. The remaining authors have disclosed that they have no financial interests, arrangements, or affiliations with the manufacturers of any products discussed in this article or their competitors.

Correspondence: Lindsey A.M. Bandini, MPH, National Comprehensive Cancer Network, 3025 Chemical Road, Suite 100, Plymouth Meeting, PA 19462. Email: bandini@nccn.org

\section{References}

1. Winchester DP. The assurance of quality care for the cancer patient. Cancer 1990;65(3 Suppl):776-779.

2. Ayanian JZ, Markel H. Donabedian's lasting framework for health care quality. N Engl J Med 2016;375:205-207

3. Rosati RJ. The history of quality measurement in home health care. Clin Geriatr Med 2009;25:121-134.

4. Department of Health and Human Services. Updates to the Quality Payment Program. Fed Regist 2019;84:40482-41289. Section III.K. Available at: https://www.govinfo.gov/content/pkg/FR-2019-08-14/pdf/ 2019-16041.pdf. Accessed November 1, 2019.

5. Oncology Care First Model: informal request for information. Available at: https://innovation.cms.gov/Files/x/ocf-informalrfi.pdf. Accessed November 13, 2019.

6. Miniemyer P. Blues plans to launch high-performance network nationwide in 2021. FierceHealthcare.com. Available at: https://www.fiercehealthcare.com/payer/blues-plans-to-launch-high-performance-networknationwide-2021. Accessed November 13, 2019

7. U.S. Department of Health and Human Services. HHS Quality Summit (QS) Fact Sheet. Available at: https://www.hhs.gov/about/news/2019/07/09/ hhs-quality-summit-fact-sheet.html. Accessed November 13, 2019.

8. Centers for Medicare \& Medicaid Services. Trump Administration's Patients Over Paperwork delivers for doctors. Available at: https://www.cms. gov/newsroom/press-releases/trump-administrations-patients-overpaperwork-delivers-doctors. Accessed December 1, 2019

9. American Cancer Society. Cancer Facts \& Figures 2015. Available at: https://www.cancer.org/research/cancer-facts-statistics/all-cancer-factsfigures/cancer-facts-figures-2015.html. Accessed October 3, 2019.

10. American College of Surgeons. CoC Quality Care Measures. Available at: https://www.facs.org/quality-programs/cancer/ncdb/qualitymeasures. Accessed December 1, 2019

11. American Society of Clinical Oncology. Don't perform surveillance testing (biomarkers) or imaging (PET, CT, and radionuclide bone scans) for asymptomatic individuals who have been treated for breast cancer with curative intent. ChoosingWisely.org. Available at: https://www.choosingwisely.org/clinician-lists/american-society-clinical-oncology-surveillancetesting-imaging-for-breast-cancer/. Accessed December 1, 2019.

12. ASCO QOPI. QOPI 2019 Reporting Tracks. Available at: https://practice. asco.org/sites/default/files/drupalfiles/QOPI-2019-Round-1-ReportingTracks-Public-Posting.pdf. Accessed November 1, 2019.

13. Quality ID \#455 (NQF 0213): percentage of patients who died from cancer admitted to the intensive care unit (ICU) in the last 30 days of life. Available at: https://qpp.cms.gov/docs/QPP_quality_measure_specifications/CQMMeasures/2019_Measure_455_MIPSCQM.pdf. Accessed December 1, 2019.

14. Department of Health and Human Services. CY 2020 Quality Reporting Requirements for Specific Providers. Fed Regist 2019;84:42044-42701. Section VIII. Available at: https://www.govinfo.gov/content/pkg/FR-201908-16/pdf/2019-16762.pdf. Accessed November 1, 2019.

15. Proportion of patients who died of cancer admitted to the ICU in the last 30 days of life. National Quality Forum. National Quality Forum Endorsed

Measures Database Website. Available at: http://www.qualityforum.org/ QPS/. Accessed December 1, 2019.

16. Quality ID \#226 (NQF 0028): preventive care and screening: tobacco use: screening and cessation intervention. Available at: https://qpp.cms.gov/ docs/QPP_quality_measure_specifications/Claims-Registry-Measures/ 2019_Measure_226_MedicarePartBClaims.pdf. Accessed December 1, 2019.

17. National Quality Forum. Preventive care and screening: tobacco use: screening and cessation intervention. Available at: http://www.qualityforum.org/QPS/0028. Accessed December 1, 2019

18. Quality ID \#453 (NQF 0210): percentage of patients who died of cancer receiving chemotherapy in the last 14 days of life. Available at: https://qpp.cms.gov/docs/QPP_quality_measure_specifications/ CQM-Measures/2019_Measure_453_MIPSCQM.pdf. Accessed December 8, 2019.

19. National Quality Forum. Proportion of patients who died of cancer receiving chemotherapy in the last 14 days of life. Available at: http://www. qualityforum.org/QPS/0210. Accessed December 1, 2019.

20. American College of Surgeons, Commission on Cancer. Oncology Medical Home Accreditation Program Standards Manual. Available at: https://www.facs.org/ /media/files/quality\%20programs/cancer/coc/ omh\%20manual.ashx. Accessed November 15, 2019.

21. National Quality Forum. Oncology: cancer stage documented. Available at: http://www.qualityforum.org/QPS/0386. Accessed December 1, 2019.

22. National Quality Forum. Proportion dying from cancer in an acute care setting. Available at: http://www.qualityforum.org/OPS/0214. Accessed December 1, 2019.

23. National Quality Forum. Proportion of patients who died of cancer not admitted to hospice. Available at: http://www.qualityforum.org/QPS/ 0215. Accessed December 1, 2019

24. Oncology Care Model. OCM performance-based payment methodology Version 3.2. Available at: https://innovation.cms.gov/Files/x/ocm-cancercodelists.pdf. Accessed December 1, 2019.

25. National Quality Forum. Prostate cancer: combination androgen deprivation therapy for high risk or very high risk prostate cancer. Available at: http://www.qualityforum.org/QPS/0390. Accessed December 1, 2019

26. Localized prostate cancer: urinary incontinence. Centers for Medicare \& Medicaid Services Measures Inventory Tool. Available at: https://cmit. cms.gov/CMIT_public/ViewMeasure?Measureld=5656. Accessed December 3, 2019.

27. Localized prostate cancer: vitality. Centers for Medicare \& Medicaid Services Measures Inventory Tool. Available at: https://cmit.cms.gov/CMIT_public/ ViewMeasure?Measureld=5657. Accessed December 3, 2019.

28. National Quality Forum. History of Prostate Cancer - Cancer Surveillance. Available at: http://www.qualityforum.org/QPS/0625. Accessed December 1, 2019.

29. McGale $\mathrm{P}$, Taylor $\mathrm{C}$, Correa $\mathrm{C}$, et al. Effect of radiotherapy after mastectomy and axillary surgery on 10-year recurrence and 20-year breast cancer mortality: meta-analysis of individual patient data for 8135 women in 22 randomised trials. Lancet 2014;383:2127-2135. 
30. Gradishar WJ, Anderson BO, Abraham J, et al. NCCN Clinical Practice Guidelines in Oncology: Breast Cancer. Version 3.2019. Accessed November 27, 2019. For the most recent version, visit NCCN.org.

31. Hahn EE, Hays RD, Kahn KL, et al. Use of imaging and biomarker tests for posttreatment care of early-stage breast cancer survivors. Cancer 2013; 119:4316-4324.

32. Khatcheressian JL, Hurley P, Bantug E, et al. Breast cancer follow-up and management after primary treatment: American Society of Clinical Oncology clinical practice guideline update. J Clin Oncol 2013;31: 961-965.

33. Seidman A, Hudis C, Pierri MK, et al. Cardiac dysfunction in the trastuzumab clinical trials experience. J Clin Oncol 2002;20:1215-1221.

34. Moja L, Tagliabue L, Balduzzi $\mathrm{S}$, et al. Trastuzumab containing regimens for early breast cancer. Cochrane Database Syst Rev 2012:CD006243.

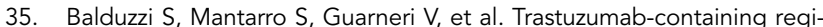
mens for metastatic breast cancer. Cochrane Database Syst Rev 2014: CD006242.

36. Henry ML, Niu J, Zhang N, et al. Cardiotoxicity and cardiac monitoring among chemotherapy-treated breast cancer patients. JACC Cardiovasc Imaging 2018;11:1084-1093.

37. Benson AB, Venook AP, Al-Hawary MM, et al. NCCN Clinical Practice Guidelines in Oncology: Colon Cancer. Version 4.2019. Accessed November 27, 2019. For the most recent version, visit NCCN.org.

38. Benson AB, Venook AP, Al-Hawary MM, et al. NCCN Clinical Practice Guidelines in Oncology: Rectal Cancer. Version 4.2019. Accessed November 27, 2019. For the most recent version, visit NCCN.org.

39. Survival Rates for Colorectal Cancer. American Cancer Society Website. Available at: https://www.cancer.org/cancer/colon-rectal-cancer/ detection-diagnosis-staging/survival-rates.html. Accessed November 27, 2019

40. Sargent D, Sobrero A, Grothey A, et al. Evidence for cure by adjuvant therapy in colon cancer: observations based on individual patient data from 20,898 patients on 18 randomized trials. J Clin Oncol 2009;27: 872-877.

41. Kavalieratos D, Corbelli J, Zhang D, et al. Association between palliative care and patient and caregiver outcomes: a systematic review and metaanalysis. JAMA 2016;316:2104-2114

42. Temel JS, Greer JA, Muzikansky A, et al. Early palliative care for patients with metastatic non-small-cell lung cancer. N Engl J Med 2010;363: 733-742.

43. Sullivan DR, Chan B, Lapidus JA, et al. Association of early palliative care use with survival and place of death among patients with advanced lung cancer receiving care in the Veterans Health Administration. JAMA Oncol 2019;5:1702

44. D'Amico AV, Chen $\mathrm{MH}$, Renshaw $\mathrm{AA}$, et al. Androgen suppression and radiation vs radiation alone for prostate cancer: a randomized trial. JAMA 2008;299:289-295

45. Denham JW, Steigler A, Lamb DS, et al. Short-term neoadjuvant androgen deprivation and radiotherapy for locally advanced prostate cancer: 10-year data from the TROG 96.01 randomised trial. Lancet Oncol 2011:12:451-459.

46. Jones CU, Hunt D, McGowan DG, et al. Radiotherapy and short-term androgen deprivation for localized prostate cancer. N Engl J Med 2011; 365:107-118

47. Ficarra $\vee$, Novara G, Ahlering TE, et al. Systematic review and metaanalysis of studies reporting potency rates after robot-assisted radical prostatectomy. Eur Urol 2012;62:418-430.

48. Chade DC, Eastham J, Graefen M, et al. Cancer control and functional outcomes of salvage radical prostatectomy for radiation-recurrent prostate cancer: a systematic review of the literature. Eur Urol 2012;61: 961-971.

49. Shekarriz B, Upadhyay J, Pontes JE. Salvage radical prostatectomy. Uro Clin North Am 2001;28:545-553.

50. Loeb S, Bruinsma SM, Nicholson J, et al. Active surveillance for prostate cancer: a systematic review of clinicopathologic variables and biomarkers for risk stratification. Eur Urol 2015;67:619-626.

51. Epstein JI, Egevad L, Amin MB, et al. The 2014 International Society of Urological Pathology (ISUP) Consensus Conference on Gleason grading of prostatic carcinoma: definition of grading patterns and proposal for a new grading system. Am J Surg Pathol 2016;40:244-252.

52. Lu-Yao GL, Albertsen PC, Moore DF, et al. Fifteen-year survival outcomes following primary androgen-deprivation therapy for localized prostate cancer. JAMA Intern Med 2014;174:1460-1467.

53. Potosky AL, Haque R, Cassidy-Bushrow AE, et al. Effectiveness of primary androgen-deprivation therapy for clinically localized prostate cancer. J Clin Oncol 2014;32:1324-1330.

54. American College of Surgeons, Commission on Cancer. Cancer Program Standards: Ensuring Patient-Centered Care. Available at: https://www. facs.org/-/media/files/quality-programs/cancer/coc/2016-coc-standardsmanual_interactive-pdf.ashx. Accessed December 1, 2019.

55. Abernethy AP, Herndon JE, Wheeler JL, et al. Poor documentation prevents adequate assessment of quality metrics in colorectal cancer J Oncol Pract 2009;5:167-174.

56. Lara PN Jr, Higdon R, Lim N, et al. Prospective evaluation of cancer clinica trial accrual patterns: identifying potential barriers to enrollment. J Clin Oncol 2001;19:1728-1733.

57. Secretan $B$, Straif $K$, Baan $R$, et al. A review of human carcinogens-part $E$ : tobacco, areca nut, alcohol, coal smoke, and salted fish. Lancet Oncol 2009; 10:1033-1034

58. Warren GW, Cummings KM. Tobacco and lung cancer: risks, trends, and outcomes in patients with cancer. Am Soc Clin Oncol Educ Book 2013;33:359-364.

59. Gritz ER, Toll BA, Warren GW. Tobacco use in the oncology setting: advancing clinical practice and research. Cancer Epidemiol Biomarkers Prev 2014;23:3-9.

60. Hanna N, Mulshine J, Wollins DS, et al. Tobacco cessation and control decade later: American Society of Clinical Oncology policy statement update. J Clin Oncol 2013;31:3147-3157.

61. Earle CC, Park ER, Lai B, et al. Identifying potential indicators of the quality of end-of-life cancer care from administrative data. J Clin Oncol 2003;21: 1133-1138.

62. Mack JW, Cronin A, Keating NL, et al. Associations between end-of-life discussion characteristics and care received near death: a prospective cohort study. J Clin Oncol 2012;30:4387-4395.

63. Institute of Medicine. Dying in America: Improving Quality and Honoring Individual Preferences Near the End of Life. Washington, DC: The National Academies Press, Institute of Medicine; 2015.

64. Kraut J, Mooney K, Sweetenham JW, et al. Implementing an electronic end-of-life chemotherapy utilization measure. J Oncol Pract 2019:15: 220-223.

65. Adelson KB, Meropol NJ, Hamrick J, et al. Development of a dashboard for end-of-life care at an academic hospital [abstract]. J Clin Oncol 2018; 36(Suppl):Abstract 6590

66. Porter ME, Larsson S, Lee TH. Standardizing patient outcomes measurement. N Engl J Med 2016;374:504-506.

67. Hassett MJ. Quality improvement in the era of big data. J Clin Onco 2017;35:3178-3180 